\title{
Mathematical modeling of ocular and cerebral hemo-fluid dynamics: application to VIIP
}

Fabrizia Salerni ${ }^{1}$, Rodolfo Repetto ${ }^{2}$, Alon Harris ${ }^{3}$, Peter Pinsky ${ }^{4}$, Christophe Prud'homme ${ }^{5}$, Marcela Szopos ${ }^{5}$, Giovanna Guidoboni ${ }^{6}$

${ }^{1}$ Mathematical, Physical and Computer Science, University of Parma, Parma, Italy; ${ }^{2}$ Department of Civil, Chemical and Environmental Engineering, University of Genoa, Genoa, Italy; ${ }^{3}$ Ophthalmology, Indiana University School of Medicine, Indianapolis, IN, USA; ${ }^{4}$ Mechanical Engineering, Stanford University, Stanford, CA, USA; ${ }^{5}$ nstitute for Advanced Mathematical Research, UMR 7501, National Center for Scientific Research, University of Strasbourg, Strasbourg, France; ${ }^{6}$ Department of Electrical Engineering and Computer Science, University of Missouri, Columbia, MO, USA

\section{Abstract}

This work aims at investigating the interactions between the flow of fluids in the brain and eyes, and their potential implications in the development of visual impairment and intracranial pressure (VIIP) syndrome in astronauts. We propose a reduced (0-D) mathematical model of fluid circulation in the eyes and brain, which is embedded into a simplified whole-body circulation model. This model allows us to predict fluid redistribution in the upper body vasculature as well as variation of the intracranial (ICP) and intraocular (IOP) pressures. The model results suggest that, by taking into account some effects of microgravity, it is possible to observe, on one hand, an increase in IOP, and on the other, a decrease in blood flow circulation in the choroid and ciliary body. These findings provide clues for the role that vascular components may play in VIIP pathogenesis, for which astronauts could be screened on Earth and in-flight.

Keywords: blood flow, intracranial pressure, intraocular pressure (IOP), visual impairment, intracranial pressure (VIIP) syndrome

Correspondence: Fabrizia Salerni, Department of Mathematical, Physical and Computer Science, University of Parma, Parco Area delle Scienze 7/A, 43124 Parma, Italy.

E-mail: fabrizia.salerni@studenti.unipr.it 


\section{Introduction}

Long-term exposure to microgravity leads to several functional changes in the human body, among which one of the most serious is loss of visual function.

The many factors hypothesized to contribute to VIIP are: upper body fluid shift, alterations in IOP, ICP, cerebrospinal fluid (CSF) pressure, tissue deformation, and blood flow. ${ }^{1}$

Due to the difficulty of singling out each of these factors using in-vivo studies, we present a novel mathematical model to evaluate the interactions between the flow and pressures of fluids in the brain (blood, CSF, and interstitial fluid) and eyes (blood and aqueous humor), and their mathematical implications in VIIP.

As suggested in the literature, the effect of microgravity is accounted for by:

1. considering zero hydrostatic pressure;

2. imposing zero central venous pressure;

3. decreasing the blood/aqueous humor oncotic pressure difference $(\Delta \pi)$; and

4. increasing the blood-brain barrier permeability. ${ }^{2}$

\section{Methods}

The physiological system is subdivided into a number of linked, interacting compartments in the brain and eyes, each of which contains a single physical constituent. An electrical analogue representation of the model is schematically shown in Figure 1.

The coupling between brain (adapted from Lakin and Stevens) ${ }^{2}$ and eye (adapted from Guidoboni et al., Szopos et al., and Kiel et al. $)^{3-5}$ models allows us to account for the following mechanisms that we believe to be relevant in VIIP:

1. blood volume changes in the brain cause variations of the ICP and affect the pressure in the retrobulbar subarachnoid space;

2. blood volume in the ocular tissue has a direct impact on IOP:

3. deformation of the lamina cribrosa influences ocular blood perfusion as a result of the combined IOP, CSF pressure, and scleral tension; and

4. increase of aqueous humor production is triggered by changes in oncotic pressures.

By writing the Kirchoff law of currents at the circuit nodes, we obtain a set of non-linear algebraic equations. The non-linearity is a consequence of the fact that, in some compartments, resistances are assumed to depend on the pressures. Following Guidoboni et al. ${ }^{3}$ and Pedley, ${ }^{6}$ we model these deformable tubes as Starling resistors, reflecting the physiological high collapsibility of these vessels when the transmural pressure becomes negative.

The model is forced by the pressure drop between the central arteries (PA) and the central veins (PV) by the active secretion of aqueous humor in the eyes and by the production of CSF in the brain. 


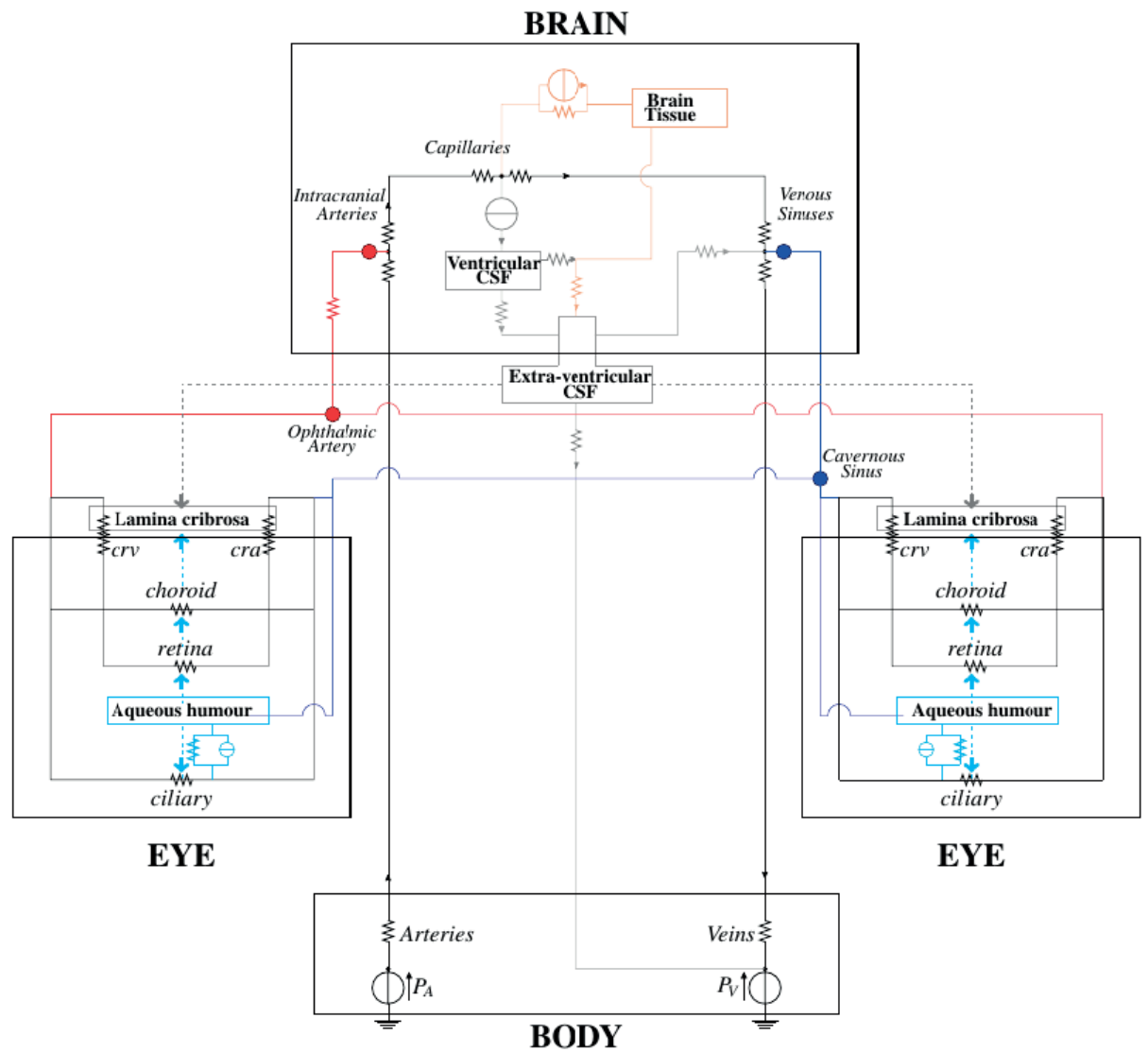

Fig. 1. 0-D model for the Body-Brain-Eyes system: blood vasculature (black portion), CSF network (grey portion), interstitial fluid network (tan portion), and aqueous humor network (cyan portion). The nodes correspond to the connection between the brain and eye models (blue arterial supply; red, venous drainage); the green and cyan arrows represent the pressures acting on both sides of the lamina cribrosa.

In this work, we focus on steady simulations, neglecting, in particular, time variations occurring on the time scale of heart beat. We also neglect autoregulation mechanisms, since we wish to keep the model relatively simple in order to understand its basic behavior. Moreover, little information exists about autoregulation mechanisms in orbit except that they might be altered due to high $\mathrm{CO}_{2}{ }^{7}$

This is the first time that all these model parts are combined into a single one. In order to assess the validity of the assumptions related to the coupling between the various components, we compared qualitatively and quantitatively the results provided by the whole model with several clinical findings: 

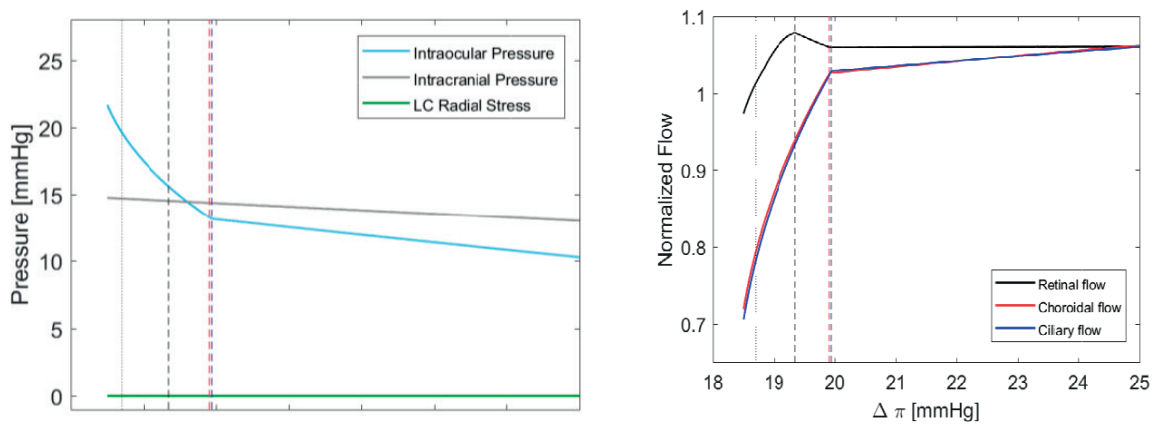

Fig. 2. (Left) Simulated IOP, ICP, and laminar compressive stress (LC radial showing that a decrease in $\Delta \pi$ in microgravity leads to a linear increase in ICP and a non-linear increase in IOP. This is due to the venous collapse: as the transmural pressure becomes negative, the Staring resistors collapse, giving rise to a significant increase in the resistance of the compartment). (Right) Simulated normalized blood flow in retinal, choroidal, and ciliary circulations. Dotted lines indicate $\Delta \pi$ values in microgravity, which differ from the physiological value on Earth of $25 \mathrm{mmHg}$. Dashed lines indicate $\Delta \pi$ values for which veins collapse in retinal (black), choroidal (red), and ciliary (blue) circulations.

1. choroidal venous pressure approximately equals IOP;

2. central retinal artery blood velocity decreases as IOP increases; and

3. IOP and ICP moderately increase with blood pressure, and the comparisons showed satisfactory agreement with experimental data.

\section{Results}

The model is used to simulate fluid shift from the limbs to the upper body, inducing changes in the plasma colloid osmotic pressures. Changes in the blood osmotic pressure modify both the brain pressure and IOP, owing to changes in the aqueous production rate.

Furthermore, the model predicts that ocular blood flow decreases markedly in the choroid and ciliary circulations when vessel collapse occurs (Fig. 2, right). Before venules collapse, the flux in the retina remains almost constant. This suggests that the particular architecture of the retinal vasculature provides a sort of mechanical (i.e., purely passive) blood flow regulation when changes in IOP occur. When venules collapse in the ciliary and choroidal circulation, the flux in the retina initially grows, and only once the central retinal veins collapse, blood flow in the retina starts to drop significantly. 


\section{Conclusion}

The model results suggest that:

1. IOP and ICP increase in microgravity conditions, but their respective trends may be different;

2. the venous segments play a fundamental role in controlling pressures and fluxes in the ocular circulation, owing to the possibility of their collapse; and

3. retinal circulation is less susceptible to microgravity-induced alterations than choroid and ciliary circulation.

These findings point towards further clinical assessment of ocular venous function in microgravity as a potential determinant factor for VIIP extensions.

\section{Acknowledgements}

This research has been partially supported by National Science Foundation (US) DMS-1224195, a grant from Research to Prevent Blindness (RPB, NY, USA), the Chair Gutenberg funds of the Cercle Gutenberg (France), and the LabEx IRMIA (University of Strasbourg, France).

\section{References}

1. Sigal IA, Flanagan JG, Tertinegg I, Ethier CR. finite element modeling in optic nerve. Invest Ophthalmol Vis Sci. 2004;45(12):4378-4387.

2. Sigal IA, Hongli Y, Roberts MD, Burgoyne CF, Downs CJ. IOP-induced lamina cribrosa displacement and scleral canal expansion: an analysis of factor interactions using parameterized eye-specific models. Invest Ophthalmol Vis Sci. 2011;52(3):1896-1907.

3. Sigal IA, Grimm JL, Jan NJ, Reid K, Minckler DS, Brown DJ. Eye-specific IOP-induced displacements and deformations of human lamina cribrosa. Invest Ophthalmol Vis Sci. 2011;55(1):1-15.

4. Causin P, Guidoboni G, Harris A, Prada D, Sacco R, Terragni S. A poroelastic model for the perfusion of the lamina cribrosa in the optic nerve head. Math Biosci. 2014;(257):33-41.

5. Guidoboni G, Harris A, Carichino L, Arieli Y, Siesky BA. Effect of intraocular pressure on the hemodynamics of the central retinal artery: a mathematical model. Math Biosci Eng. 2014;(11):523-546.

6. Biot MA. General theory of of three-dimensional consolidation. J Appl Phys. 1941;(12):155-164.

7. Biot MA. Theory of finite deformations of porous solids. Ind Univ Math J. 1972;21:597-620.

8. Larchè F, Cahn JW. Linear theory of thermomechanical equilibrium solids under stress. Acta. Metall. 1973;21:1051-1063.

9. Larchè F, Cahn JW. The interactions of composition and stress in crystalline solids. J Res Natl Bur Stand. 1984;89:467-500.

10. Honga W, Zhaoa X, Zhoua J, Suo Z. A theory of coupled diusion and large deformation in polymeric gels. J Mechan Phys Solids. 2008;56:1779-1793.

11. Eshelby JD. Elastic energy momentum tensor. J Elasticity. 1975;5:331-335. 